\title{
Inhibition of integrin $\beta 1$ decreases the malignancy of ovarian cancer cells and potentiates anticancer therapy via the FAK/STAT1 signaling pathway
}

\author{
LEI ZHANG ${ }^{1}$ and WEN ZOU ${ }^{2}$ \\ Departments of ${ }^{1}$ Urology and ${ }^{2}$ Oncology, \\ The Second Xiangya Hospital of Central South University, Changsha, Hunan 410011, P.R. China
}

Received October 18, 2014; Accepted June 26, 2015

DOI: $10.3892 / \mathrm{mmr} .2015 .4443$

\begin{abstract}
Integrin $\beta 1$ (ITGB1) is frequently upregulated in ovarian cancer, and promotes ovarian tumorigenesis and cancer progression. However, the effects of ITGB1 inhibition on ovarian cancer progression and anticancer therapy remain to be elucidated. The results of the present study indicated that ITGB1 was upregulated in HO-8910 and HO-8910PM ovarian cancer cell lines, and knockdown of ITGB1 using short hairpin RNA markedly increased tumor cell apoptosis, decreased tumor cell adhesion and migration, and reduced tumor cell invasion by suppressing matrix metalloproteinase (MMP)-2 and MMP-9 expression. Furthermore, the results of the present study provided evidence regarding the role of ITGB1 inhibition in bevacizumab anticancer therapy. The activation of signal transducer and activator of transcription 1 (STAT1) by focal adhesion kinase (FAK) is involved in integrin-mediated cell migration and adhesion. In the present study, the expression levels of FAK were markedly upregulated in ovarian cancer. The adherence and migratory potentials of ovarian cancer cells were significantly reduced when the FAK/STAT1 signaling pathway was inhibited by fludarabine. The results of the present study demonstrated that ITGB1 inhibition effectively reduced tumorigenesis and disease exacerbation, and contributed to bevacizumab anticancer therapy via the FAK/STAT1 signaling pathway, suggesting that inhibition of ITGB1 is a potential novel therapeutic strategy for ovarian carcinogenesis.
\end{abstract}

Correspondence to: Dr Wen Zou, Department of Oncology, The Second Xiangya Hospital of Central South University, 139 Renmin Road, Changsha, Hunan 410011, P.R. China E-mail: csuzouwen2014@163.com

Key words: integrin $\beta 1$, bevacizumab, focal adhesion kinase/signal transducer and activator of transcription pathway, ovarian cancer

\section{Introduction}

Ovarian cancer is the most lethal gynecological malignancy, and one of the leading causes of morbidity and mortality worldwide (1). In ovarian carcinoma, cancer cells frequently metastasize by implanting onto the peritoneal mesothelial surface of the abdominal cavity. The resulting peritoneal implants are characterized by the adhesion, migration, and invasion of the tumor cells into the peritoneum and underlying organs (2).

Integrins are a large family of cell surface adhesion proteins that are involved in epithelial cell-matrix interactions. Upregulation of integrins has been associated with malignancy $(3,4)$, particularly during invasion, metastasis, and angiogenesis $(5,6)$. Increasing evidence suggests that aberrantly expressed integrins have a role in ovarian cancer pathophysiology (7-9). Integrin $\beta 1$ (ITGB1), regulates broader functional activities such as cellular proliferation, adhesion, and invasion, and previous studies have suggested its implication in therapeutic resistance in numerous solid cancer models (10-12) and hematopoietic malignancies $(13,14)$. Focal adhesion kinase $($ FAK) is upregulated in various malignancies, such as breast, colon, prostate, neck, and ovarian cancer $(15,16)$. FAK is an important regulator of survival, proliferation, migration, and invasion, processes that are all involved in tumorigenesis and metastasis $(17,18)$. Therefore, FAK signaling may be regarded as a potential target in the development of anticancer drugs. A previous study demonstrated that FAK, identified as a downstream kinase of ITGB1, has an important role in integrin-stimulated signaling events. Integrin clustering induces autophosphorylation of FAK and activates signaling pathway networks (19). However, the mechanisms underlying ITGB1 inhibition-induced suppression of ovarian cancer progression, and the contribution of ITGB1 inhibition in anticancer therapy remains to be elucidated. The present study investigated the effects of ITGB1 inhibition on tumor apoptosis, invasion and migration, and bevacizumab anticancer therapy in ovarian cancer cells. Furthermore, the present study investigated the signaling pathways that may mediate the anti-invasive and anti-migratory effects that resulted from ITGB1 inhibition. 


\section{Materials and methods}

Ovarian cancer cell culture. Human ovarian surface epithelial cells (HOSEpic), HO-8910 and HO-8910PM ovarian cancer cells were used in the present study.HO-8910PM cells are highly metastatic ovarian cancer cells derived from HO-8910 (20). The cell lines were grown in RPMI 1640 (GE Healthcare Life Sciences, Hudson, NH, USA) supplemented with $10 \%$ fetal bovine serum (Invitrogen Life Technologies, Carlsbad, CA, USA), $1 \%$ penicillin and streptomycin (Sigma-Aldrich, St. Louis, MO, USA), in an atmosphere containing $5 \% \mathrm{CO}_{2}$, at $37^{\circ} \mathrm{C}$. The cell lines were purchased from the Cell Bank of Shanghai Institutes for Biological Sciences, the Chinese Academy of Science (Shanghai, China).

RNA extraction and reverse transcription-quantitative polymerase chain reaction (RT- $q P C R)$. Total RNA was extracted from the cells with TRIzol (Invitrogen Life Technologies) and reverse transcribed into cDNA with reverse transcriptase from the Primescript RT Reagent kit (Takara Biotechnology Co., Ltd., Dalian, China), as described previously (21). The RT-qPCR reactions were performed using the Thermal Cycler Dice Real-Time PCR system (Takara Biotechnology Co., Ltd.) and the ABI 7300 Thermal Cycler (Applied Biosystems Life Technologies, Carlsbad, CA, USA) with the following cycling conditions: Initial denaturation at $95^{\circ} \mathrm{C}$ for $10 \mathrm{~min}$, and 40 cycles of $95^{\circ} \mathrm{C}$ for $30 \mathrm{sec}, 55^{\circ} \mathrm{C}$ for $30 \mathrm{sec}$, and $72^{\circ} \mathrm{C}$ for $2 \mathrm{~min}$. In order to ensure the reproducibility of the results, all the genes were tested in triplicate. The following primers from BGI Tech (Shenzhen, China) were used for the present study: ITGB1, sense 5'-GTCTCAGACTGGCTTCAGTG-3', antisense 5'-ATGATTGTACCGAGGCTGTC-3'; FAK, sense 5'-GCGGCCCAGGTTTACTGAA-3', antisense 5'-GGCCTGTCTTCTGGACTCCAT- 3 '; and $\beta$-actin (human), sense 5'-AGGGGCCGGACTCGTCATACT-3', and antisense 5'-GGCGGCACCACCATGTACCCT-3'. Reaction of each sample was performed in triplicate. Relative expression levels were calculated using the $2-\Delta \Delta \mathrm{Ct}$ method.

Lentivirus packaging and infection. Three precursor small hairpin RNA sequences targeting ITGB1 (GenBank accession no. NM_133376; GTGTACAGATCCGAAGTTTCA) were designed using an Internet application system (http://rnaidesigner.lifetechnologies.com/rnaiexpress/plate.jsp; Invitrogen Life Technologies). The anti-ITGB1 sequence was inserted into a lentiviral vector gene (Shanghai GenePharma Co., Ltd., Shanghai, China) in order to construct a recombinant lentiviral vector GV248, as previously described (22). HO-8910 and HO-8910PM cells in 6-well plates $\left(2 \times 10^{5}\right.$ cells/well) were transfected with $42 \mu 1$ ITGB1-shRNA lentiviral vector (LV; $2.40 \times 10^{8}$ transducing units) or $12 \mu 1$ negative control (NC) lentiviral vector (LV-NC; $1.8 \times 10^{9}$ transducing units) at $37^{\circ} \mathrm{C}$. A total of $5 \mu \mathrm{g} / \mathrm{ml}$ polybrene (Sigma-Aldrich) was added to enhance the transfection efficiency. A selection medium supplemented with $2.5 \mu \mathrm{g} / \mathrm{ml}$ puromycin (Sigma-Aldrich) was used to obtain a stable transfected cell line over the course of 2 weeks.

Treatment of cells with bevacizumab and fludarabine. The ovarian cancer cells transfected with the LV-ITGB1-RNAi vector were treated with $10 \mu \mathrm{g} / \mathrm{ml}$ bevacizumab (Avastin ${ }^{\circledR}$; Genentech, Inc., South San Francisco, CA, USA) for 24 h, and then cell apoptosis, adhesion and invasion were detected. The HO-8910 and HO-8910PM cells were treated with $1.54 \mu \mathrm{g} / \mathrm{ml}$ fludarabine (Selleck Chemicals, Houston, TX, USA) for $24 \mathrm{~h}$, and then protein expression, adhesion and invasion were detected.

Western blot analysis. Whole cell extracts were prepared using radioimmunoprecipitation assay lysis reagent (Sigma-Aldrich), according to the manufacturer's instructions. The proteins were then quantified using a bicinchoninic acid assay (Pierce Biotechnology, Inc., Rockford, IL, USA), prior to being separated by $10 \%$ SDS-PAGE, and detected by western blotting on a polyvinylidene difluoride membrane (EMD Millipore, Billerica, MA, USA). The membranes were blocked with $3 \mathrm{ml}$ Tris-buffered saline with Tween 20 (TBST; Auragene Bioscience Co., Changsha, China) with $5 \%$ bovine serum albumin (Auragene Bioscience Co.) for $2 \mathrm{~h}$ at room temperature. The membranes were incubated overnight at $4^{\circ} \mathrm{C}$ with the following primary antibodies: Rabbit polyclonal anti-ITGB1 (sc-13590; 1:200), anti-STAT1 (sc-345; 1:500), anti-phosphorylated STAT1 (sc-135648; 1:500), anti-FAK (sc-558; 1:500), anti-matrix metalloproteinase (MMP)-2 (sc-13594; 1:500) and MMP-9 (sc-21733; 1:200) primary antibodies (Santa Cruz Biotechnology, Inc., Dallas, TX, USA). The membranes were incubated for $1 \mathrm{~h}$ at $37^{\circ} \mathrm{C}$ with the anti- $\beta$-actin antibody (sc-8979; $1: 6,000$; Santa Cruz Biotechnology, Inc.). The membranes were washed 3 times with TBST, then incubated with the horseradish peroxidase-conjugated goat anti-rabbit immunoglobulin G secondary antibody (Pierce Biotechnology, Inc.), followed by 3 additional washes with TBST. A SuperSignal West Femto Enhanced Chemiluminescence Detection system (Pierce Biotechnology, Inc.) was used for detection. The blots were analyzed with the CanoScan LiDE 110 scanner (Canon, Inc., Tokyo, Japan) and Image Pro-Plus software, version 6.0 (Media Cybernetics, Inc., Rockville, MD, USA).

In vitro adhesion assay. The cells were pretreated with or without bevacizumab $(10 \mu \mathrm{g} / \mathrm{ml})$ for $24 \mathrm{~h}$. The cells were then suspended in serum-free Dulbecco's modified Eagle's medium (GE Healthcare Life Sciences) to form a single-cell suspension, prior to being seeded at a density of $10^{5}$ cells/well into 96-well plates precoated with Matrigel ${ }^{\mathrm{TM}}$ (BD Biosciences, Franklin Lakes, NJ, USA). The wells were incubated at $37^{\circ} \mathrm{C}$ for $50 \mathrm{~min}$, and washed three times with phosphate-buffered saline (PBS) in order to remove non-adherent cells. Cell viability was determined using an MTT assay.

Cell survival assay. An MTT assay was used to estimate cell viability (23). Briefly, the cells were plated at a density of $1 \times 10^{4}$ cells/well in 96-well plates. Following exposure to the original and the stably lentivirus infected cell lines of ovarian cancer, with or without bevacizumab $(10 \mu \mathrm{g} / \mathrm{ml})$ treatment for $24 \mathrm{~h}$, the cells were incubated with MTT (Beijing Solarbio Science \& Technology Co., Ltd., Beijing, China) at a final concentration of $0.5 \mathrm{mg} / \mathrm{ml}$ for $4 \mathrm{~h}$ at $37^{\circ} \mathrm{C}$. The medium was carefully discarded, and $150 \mathrm{mM}$ dimethyl sulfoxide (Beijing Solarbio Science \& Technology Co., Ltd.) 
A
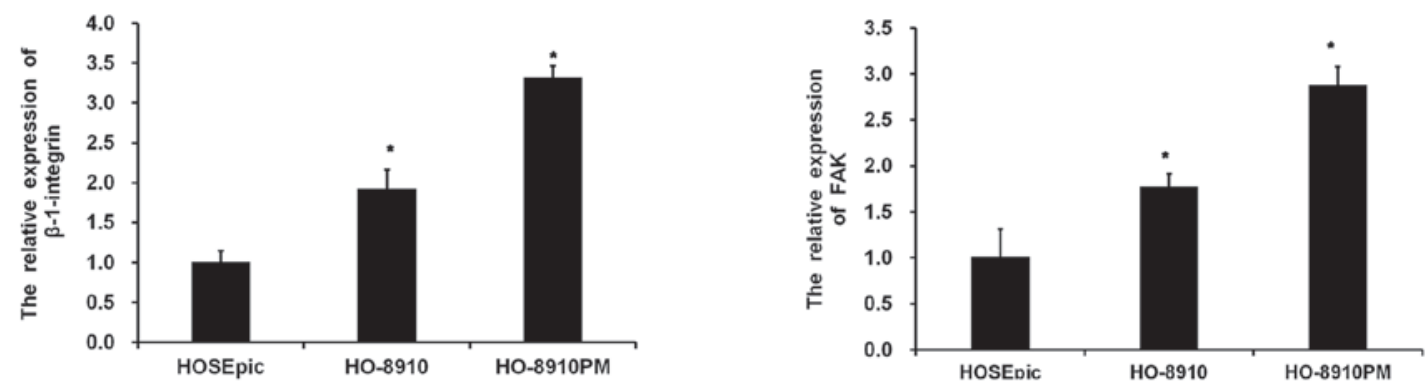

B
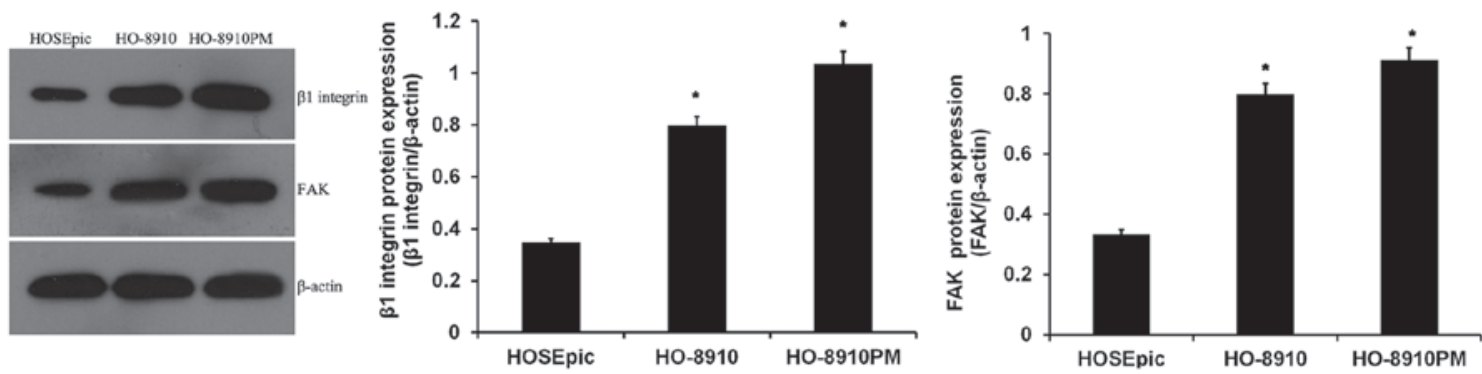

Figure 1. Expression of integrin $\beta 1$ (ITGB1) and focal adhesion kinase (FAK) in ovarian cancer cells. (A) Reverse transcription-quantitative polymerase chain reaction detected the mRNA expression levels of ITGB1 and FAK in normal human ovarian surface epithelial cells (HOSEpic), and ovarian cancer cells (HO-8910 and HO-8910PM). (B) Western blot analysis detected the protein expression levels of ITGB1 and FAK. Data are presented as the mean \pm standard deviation. ${ }^{*} \mathrm{P}<0.01$, vs. the normal ovarian cells (HOSEpic).

was added to dissolve the formazan crystals. The absorbance was measured at $570 \mathrm{~nm}$ using a multi-well scanning spectrophotometer reader (756MC; Jinghua Instruments, Shanghai, China). The cells in the control group were considered $100 \%$ viable.

Flow cytometry. An Annexin V and propidium iodide (PI) Fluorescein Staining kit (Bender MedSystems GmbH, Wien, Austria) were used to measure cell apoptosis, according to the manufacturer's instructions. Briefly, $5 \times 10^{5}$ cells were suspended in $500 \mu \mathrm{l} 1 \mathrm{X}$ binding buffer containing $10 \mathrm{mM}$ HEPES (pH 7.4), $140 \mathrm{mM} \mathrm{NaCl}$, and $2.5 \mathrm{mM} \mathrm{CaCl}_{2}$. The cells were then incubated with annexin $\mathrm{V}(1: 20)$ for $5 \mathrm{~min}$, prior to being incubated with PI $(1 \mathrm{mg} / \mathrm{ml})$ for $15 \mathrm{~min}$. The rate of apoptosis was evaluated by flow cytometry $\left(\mathrm{MoFlo}^{\mathrm{TM}} \mathrm{XDP}\right.$; Beckman Coulter, Inc., Brea, CA, USA).

Invasion assay. The cells were cultivated to $80 \%$ confluence in 12 -well plates. Cellular growth was subsequently observed for $24 \mathrm{~h}$. All the experiments were repeated in triplicate. Cell invasion was determined by the Transwell assay. Cells suspended in serum-free medium were added into the upper chamber of the insert with Matrigel (BD Biosciences). Following incubation for $24 \mathrm{~h}$ at $37^{\circ} \mathrm{C}$, cells remaining on the upper side of the membrane were carefully removed, while cells that had migrated through the membrane were fixed with $75 \%$ alcohol (Auragene Bioscience Co.) and stained with crystal violet (Sigma-Aldrich) for $25 \mathrm{~min}$, washed with water and air-dried. The cells that invaded the membrane were counted using a light microscope (AE31; Motic, Xiamen, China).

Migration assay. For the wound healing assay, cells were seeded in 12 -well plates and grown to $90 \%$ confluence. Monolayers in the center of the wells were scraped with pipette tips and washed with PBS. Cell movement into the wound area was monitored and the images were captured at 0 and $24 \mathrm{~h}$ using the AE31 light microscope. The migration distance between the leading edge of the migrating cells and the edge of the wound was compared, as previously described (24).

Statistical analysis. Each experiment was repeated in triplicate. Data are presented as the mean \pm standard deviation, and analyzed using SPSS 18.0 (SPSS, Inc., Chicago, IL, USA). Statistical comparisons between the groups were analyzed using two-tailed Student's t-tests. $\mathrm{P}<0.05$ was considered to indicate a statistical significant difference.

\section{Results}

Expression levels of ITGBI and FAK in ovarian cancer cells. To assess whether ITGB1 and FAK were dysregulated in ovarian cancer cells, the expression levels of ITGB1 and FAK in normal ovarian cells (HOSEpic) and ovarian cancer cells (HO-8910 and HO-8910PM) were analyzed. As shown in Fig. 1A, the mRNA expression levels of ITGB1 and FAK were significantly increased in metastatic ovarian cancer cell lines, as compared with the normal ovarian cells, with the expression levels of ITGB1 and FAK being the highest in the highly metastatic HO-8910PM cell line. Similar results were obtained from the western blot analysis (Fig. 1B). These data suggest that suppression of ITGB1 may be associated with carcinogenesis of ovarian cancer.

ITGB1 inhibition suppresses cell invasion and MMP expression in ovarian cancer cells. Previous studies have demonstrated that ITGB1 may mediate tumor growth, apoptosis, adhesion, and signaling in various cancer cells $(7,22)$. 
A
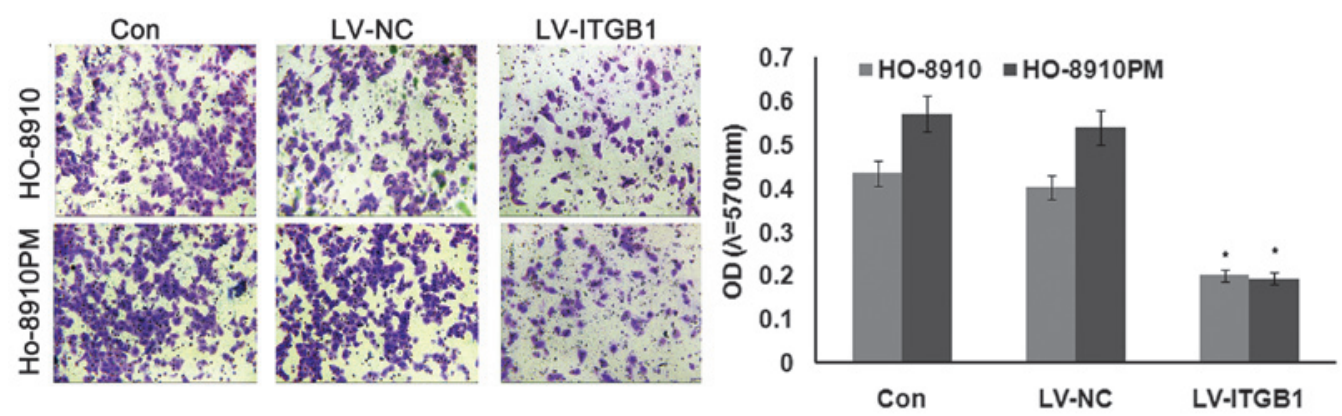

B
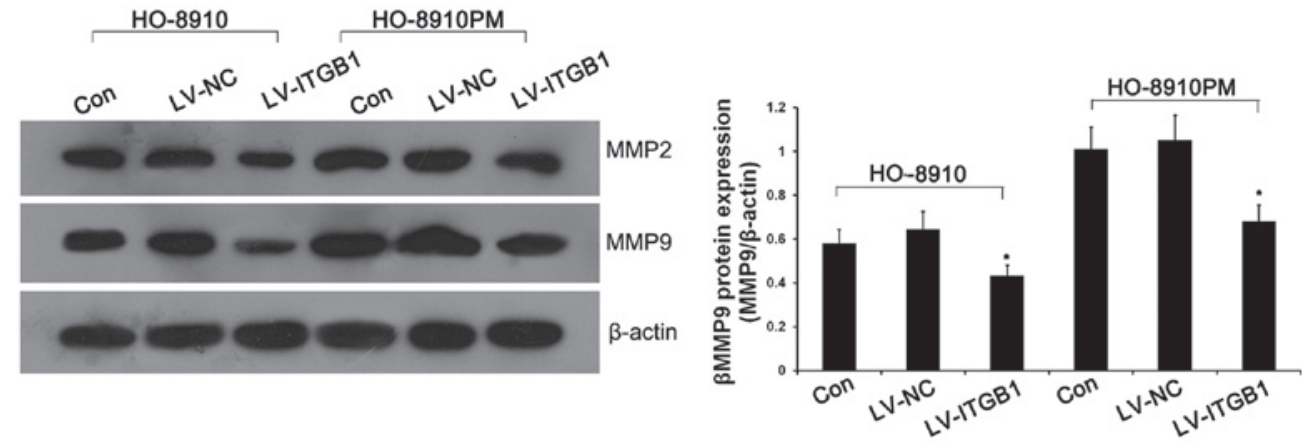

Figure 2. Integrin $\beta 1$ (ITGB1) inhibition suppresses invasion and expression of matrix metalloproteinase (MMP)-2 and MMP-9 in ovarian cancer. (A) Transwell invasion chambers were used to evaluate cell invasion. Representative photomicrographs of the membrane-associated cells were assayed by Giemsa staining (magnification, x200). (B) Western blot analysis was used to determine the effects of ITGB1 inhibition on MMP-2 and MMP-9 expression. Data are presented as the mean \pm standard deviation of duplicates from a representative experiment performed in triplicate. "P<0.01, vs. the control (Con) and lentiviral vector (LV)-NC groups.

A

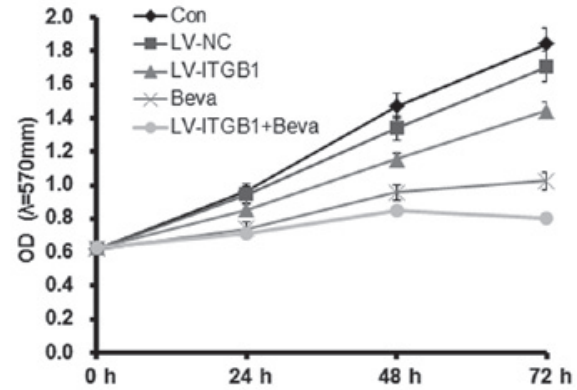

B

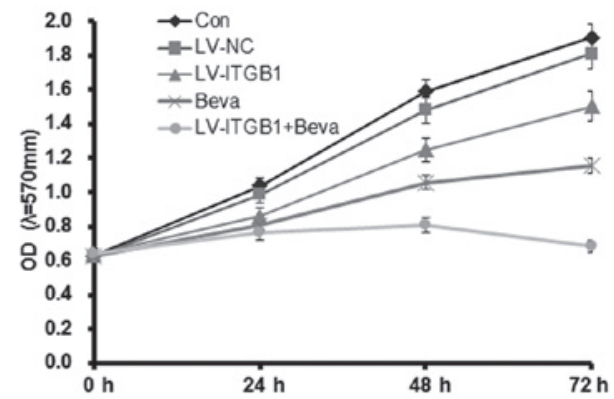

C
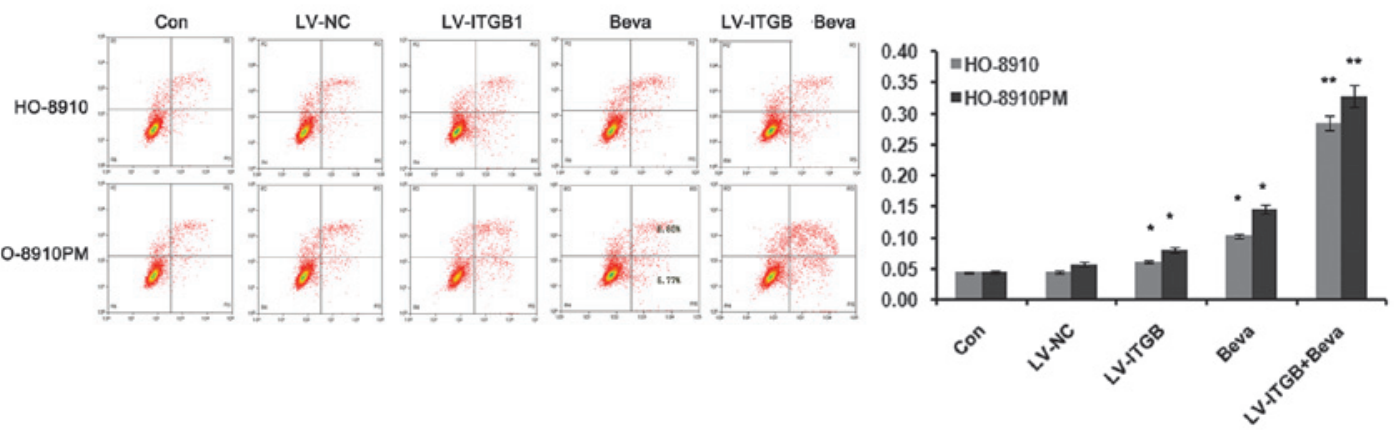

Figure 3. Integrin $\beta 1$ (ITGB1) inhibition enhances the effect of bevacizumab treatment on cell apoptosis. (A and B) MTT analysis. (C) Flow cytometric assay. Data are presented as the mean \pm standard deviation of duplicates from a representative experiment performed in triplicate. ${ }^{*} \mathrm{P}<0.01$, vs. the control (Con) and lentiviral vector (LV)-negative control (NC) groups. ${ }^{* *} \mathrm{P}<0.01$, vs. the LV-ITGB1 and bevacizumab-treated (Beva) groups.

Due to the fact that invasion is an important factor for cancer expansion and metastasis, the present study examined whether ITGBI inhibition altered the rate of invasion of cancer cells through transwell chambers coated with Matrigel ${ }^{\mathrm{TM}}$. Anti-ITGB1 lentiviral vectors were transfected into HO-8910 and HO-8910PM cells to inhibit the expression of ITGB1. Concordant with the previous results, significant attenuation of ovarian cancer cell invasion was observed in the lentivirus (LV)-ITGB1-RNA interference (RNAi) group, as compared with the control and LV-NC groups (Fig. 2A). 

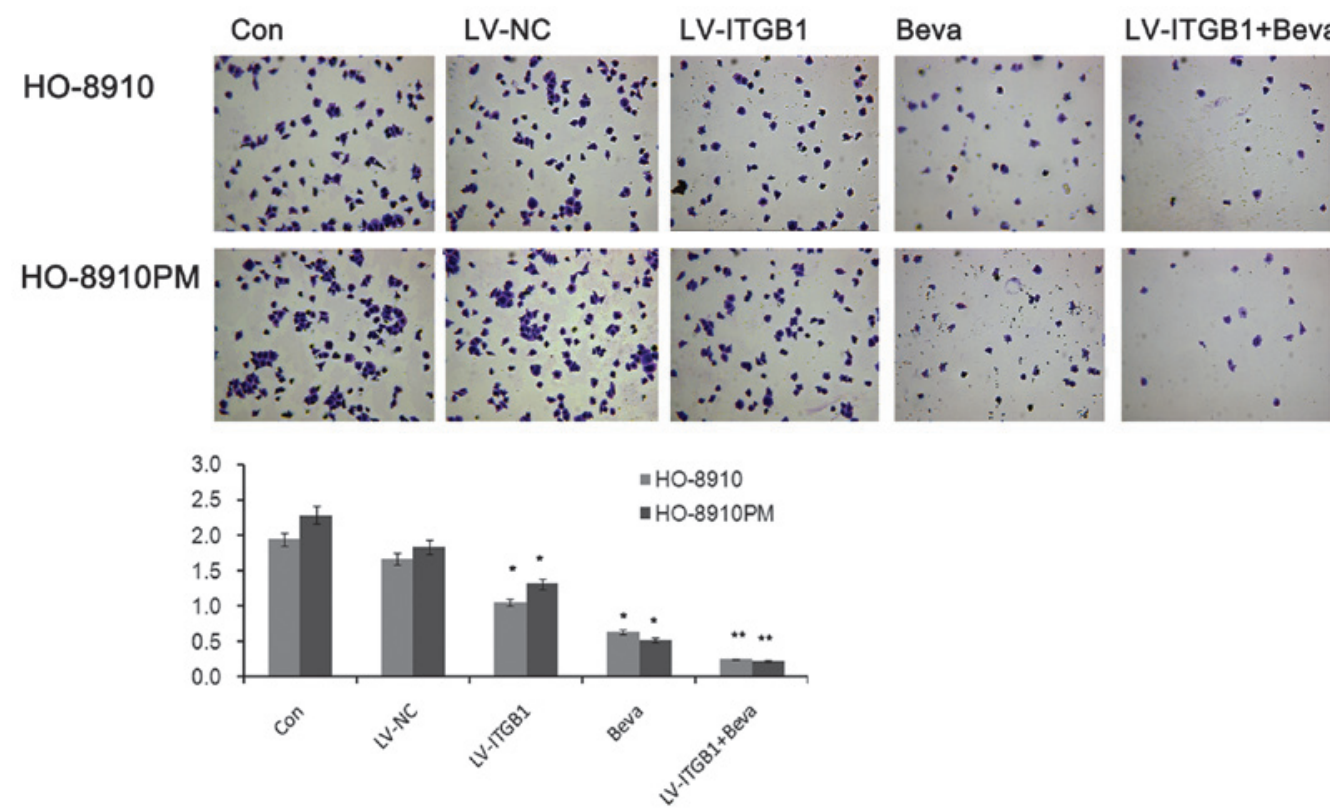

Figure 4. Effects of integrin $\beta 1$ (ITGB1) inhibition and bevacizumab treatment on ovarian cancer cell adhesion. Data are presented as the mean \pm standard deviation of duplicates from a representative experiment performed in triplicate. ${ }^{*} \mathrm{P}<0.01$, vs. the control (Con) and lentiviral vector (LV)-negative control (NC) groups. ${ }^{* *} \mathrm{P}<0.01$, vs. the LV-ITGB1 and bevacizumab-treated (Beva) groups.
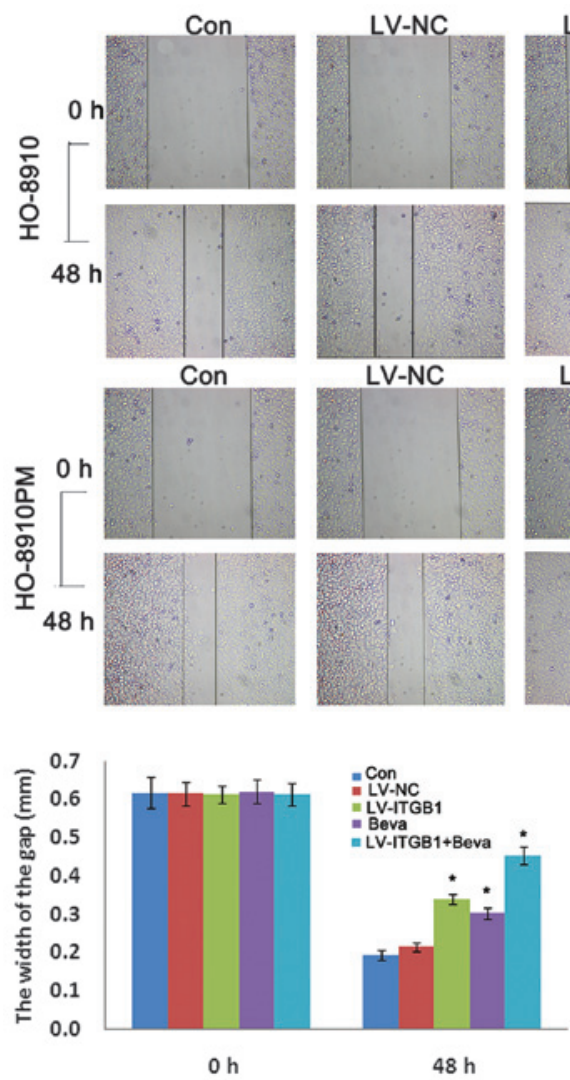
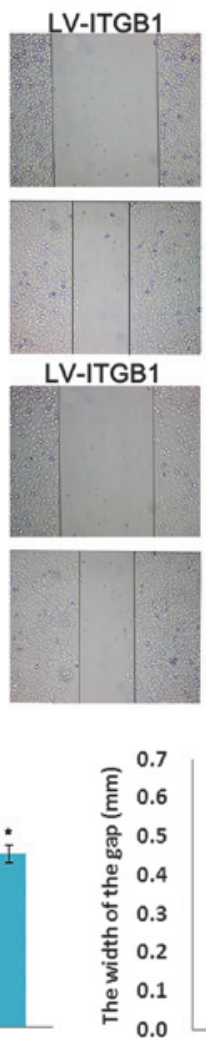

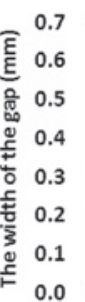

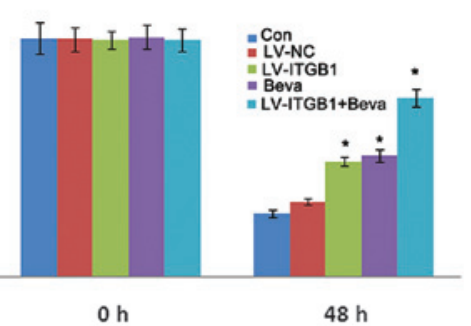

Figure 5. Effects of integrin $\beta 1$ (ITGB1) inhibition and bevacizumab treatment on ovarian cancer cell invasion, as determined by wound healing assay. A wound was introduced by scraping a pipette tip across confluent HO-8910 and HO-8910PM cells. After 24 and 48 h, images of the cells were captured under phase-contrast microscopy (magnification, $\mathrm{x} 40$ ). Data are presented as the mean \pm standard deviation of duplicates from a representative experiment performed in triplicate. ${ }^{*} \mathrm{P}<0.05$, vs. the control (Con) and lentiviral vector (LV)-negative control (NC) groups.

MMPs, specifically MMP-2 and MMP-9, are the most important proteases for the degradation of type IV collagen, which is the predominant constituent of extracellular matrix
(ECM) $(25,26)$, and has an important role in cancer cell metastasis. In order to determine the effects of ITGB1 inhibition on MMP-2 and MMP-9 protein expression, western 
A
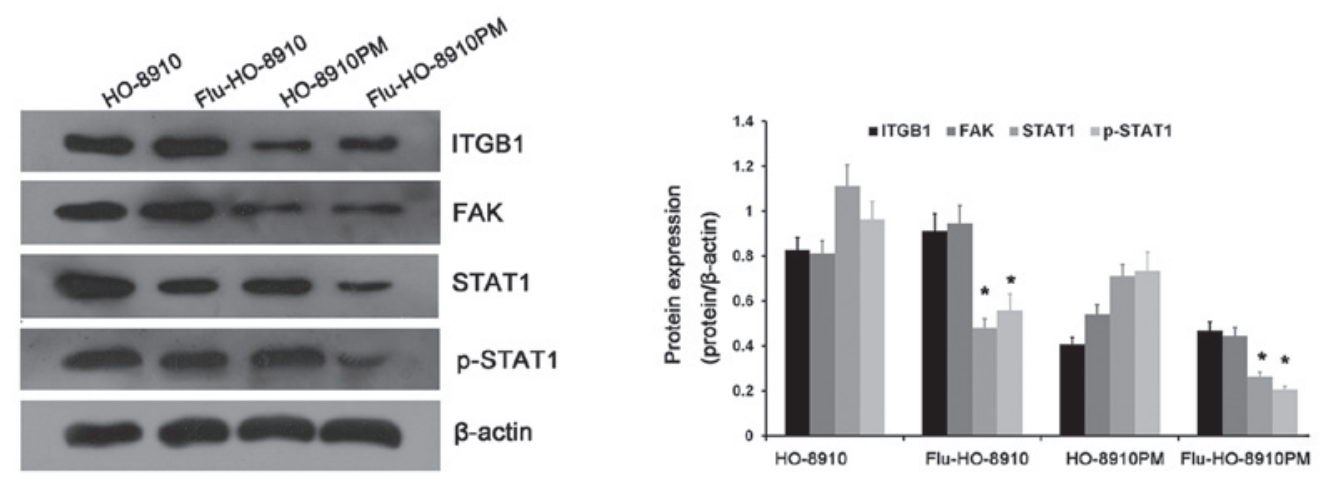

B
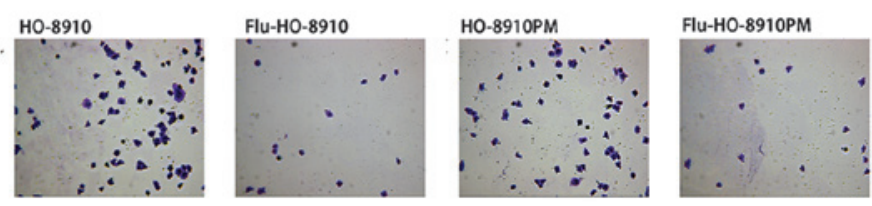

C
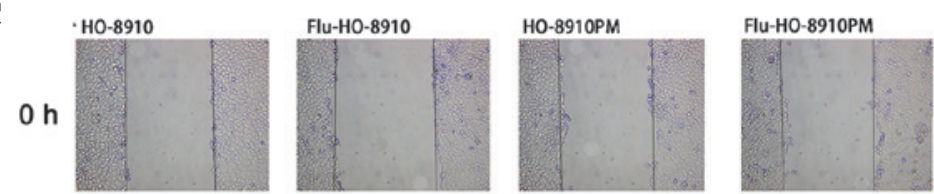

$48 \mathrm{~h}$
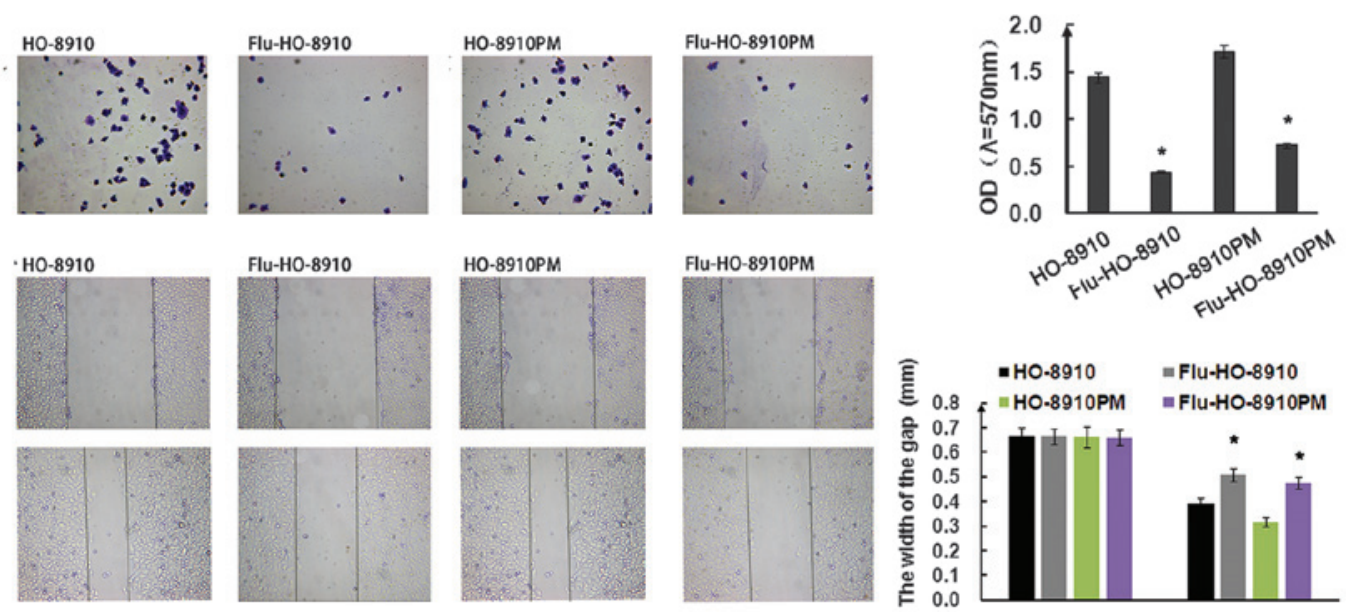

Oh

$48 \mathrm{~h}$

Figure 6. Integrin $\beta 1$ (ITGB1)/focal adhesion kinase (FAK)/signal transducer and activator of transcription 1 (STAT1) pathway regulates cell adherence and migration in ovarian cancer. (A) The effects of fludarabine (Flu) on the ITGB1/FAK/STAT1 pathway. HO-8910 and HO-8910PM cells were treated with $1.54 \mu \mathrm{g} / \mathrm{ml}$ Flu for $24 \mathrm{~h}$, prior to being collected and lysed. Total protein was separated by SDS-PAGE and analyzed by immunoblotting with the indicated antibodies. The results are representative of three different experiments. The effects of Flu on (B) adhesion and (C) migration of ovarian cancer cells. Data are presented as the mean \pm standard deviation of experiments performed in triplicate. ${ }^{*} \mathrm{P}<0.01$, vs. control. Similar results were obtained in two additional experiments.

blotting was performed. As shown in Fig. 2B, ITGB1 knockdown significantly downregulated the protein expression levels of MMP-2 and MMP-9 in the ITGB1-RNAi group, as compared with the control and LV-NC groups. These results suggest that ITGB1 inhibition suppresses MMP-2 and MMP-9 expression.

ITGB1 inhibition enhances the effect of bevacizumab treatment. Bevacizumab offers promise as an antiangiogenic in the treatment of ovarian cancer (27); however, acquired resistance limits the use of bevacizumab as a therapeutic agent. The present study demonstrated the effects of the combination of ITGB1 inhibition and bevacizumab treatment on apoptosis, adhesion and migration of ovarian cancer cells. Ovarian cancer cells with knocked down ITGB1 were treated with $10 \mu \mathrm{g} / \mathrm{ml}$ bevacizumab for $24 \mathrm{~h}$, and cell apoptosis, adhesion, and invasion were detected.

As shown in Fig. 3, MTT assay and flow cytometry were used to detect HO-8910 and HO-8910PM cell apoptosis. Cellular proliferation markedly decreased following ITGB1 inhibition and treatment with $10 \mu \mathrm{g} / \mathrm{ml}$ bevacizumab (Fig. 3A and B). Furthermore, ITGB1 inhibition further reduced cell proliferation in bevacizumab-treated ovarian cancer cells. Concordant with the results of the MTT assay, Annexin V/PI staining demonstrated that the percentage of apoptotic cells was significantly increased in both the LV-ITGB1 and bevacizumab (Beva) groups, as compared with the control group. In addition, ITGB1 inhibition further increased cell apoptosis in bevacizumab-treated ovarian cancer cells. These results suggest that ITGB1 inhibition regulates bevacizumab therapy by decreasing cell proliferation and increasing cell apoptosis.

The adhesion of cancer cells to endothelial cells or the ECM has an important role in establishing metastasis (20). Integrin regulates adhesion and signaling in ovarian cancer (7). The present study evaluated the effects of ITGB1 silencing on ovarian cancer cell adhesion to ECM proteins. The number of adherent cells markedly decreased following ITGB1 inhibition (Fig. 4). The inhibitory effects of cell adhesion induced by ITGB1 silencing may contribute to the inhibition of cell metastasis. Concordant with the results of the apoptosis analysis, ITGB1 inhibition enhanced the effects of bevacizumab treatment against cell adhesion in ovarian cancer. As shown in Fig. 4, significant attenuation of ovarian cancer cell adhesion was observed in the LV-ITGB1 + Beva group, as compared with the Beva group.

The inhibitory effects of ITGB1 suppression on ovarian cancer cell migration were determined by wound healing assay. HO-8910 and HO-8910PM cells were transfected with 
ITGB1-RNAi and treated with bevacizumab, and wound closure was monitored and the images of the wound closure were captured. The results of the wound healing assay indicated that ITGB1 inhibition significantly attenuated HO-8910 and HO-8910PM cell migration after $48 \mathrm{~h}$ (Fig. 5). These data indicate that ITGB1 silencing may effectively inhibit HO-8910 and HO-8910PM cell motility. Furthermore, cell migration was significantly decreased in the LV-ITGB1 + Beva group, as compared with the Beva group. These data suggest that the the inhibitory effects of combination treatment of bevacizumab and ITGB1 have an improved effect compared with bevacizumab simple treatment alone on HO-8910 and HO-8910PM cell migration.

ITGBI/FAK/STAT1 pathway in ovarian cancer cells. The subsequent experiments of the present study focused on the signaling pathways involved in ITGB1-mediated suppression of metastatic potential in ovarian cancer cells. To determine whether the STAT1 signaling pathway was involved in cell adhesion and migration in ovarian cancer, HO-8910 and HO-8910PM cells were treated with $1.54 \mu \mathrm{g} / \mathrm{ml}$ fludarabine for $24 \mathrm{~h}$. ITGB1 and FAK expression were not significantly affected by fludarabine. However, STAT1 and p-STAT1 expression were markedly downregulated by fludarabine (Fig. 6A). As shown in Fig. 6B, the adhesion ratio was significantly decreased in the fludarabine group, as compared with the control group. Concordant with the adhesion assay results, fludarabine significantly inhibited ovarian cancer cell migration, as determined by the wound healing assay (Fig. 6C). FAK and c-Src form a dual kinase complex, which functions to promote cell motility, cell cycle progression, and cell survival. Previous studies have demonstrated that ITGB1-mediated FAK signaling is closely associated with tumor growth and metastasis $(19,28)$. In a previous study, co-immunoprecipitation and in vitro binding assays demonstrated that STAT1 was transiently and directly associated with FAK during cell adhesion (29), and its activity was induced by the integrin signaling pathway. These results indicate that the ITGB1/FAK/STAT1 pathway is involved in cell adhesion and migration in ovarian cancer.

\section{Discussion}

Integrin-mediated cell adhesion and migration have essential roles in cell growth and development. Previous studies have demonstrated that ITGB1 is able to mediate ovarian carcinoma cell adhesion, invasion, and migration $(8,30)$. In the present study, the anti-metastatic effects of ITGB1 inhibition on the HO-8910 and HO-8910PM ovarian cancer cell lines, as well as its molecular mechanism of action, were investigated. ITGB1 inhibition induced cell apoptosis, which was determined by the inhibition of cell adhesion, migration, and invasion, as well as by the suppression of MMP-2 and MMP-9 expression. The results of the present study also demonstrated that ITGB1 inhibition enhanced bevacizumab treatment in ovarian cancer. Furthermore, the inhibition of STAT1 signaling by fludarabine revealed that the ITGB1/FAK/STAT1 pathway may be associated with the molecular mechanisms that underlie the anti-invasive effects of ITGB1 inhibition.
Metastasis is closely associated with cancer therapeutic efficacy and patient prognosis. Metastasis is a multistep process involving numerous factors. Cellular migration, the attachment of cancer cells to the ECM components, and invasion into surrounding tissues are critical to metastasis. Therefore, decreased migration, cell-matrix adhesion, and invasive potential may contribute to the prevention of metastasis. In the present study, the effects of ITGB1 inhibition on apoptosis, migration, invasion, and adhesion to ECM proteins were determined. The results indicated that ITGB1 inhibition significantly increased cell apoptosis, as determined by flow cytometry, and suppressed the migration and invasion of ovarian cancer cells, as determined by wound healing and transwell invasion assays. The cell adhesion assay revealed that inhibition of ITGB1 attenuated the adhesion of ovarian cancer cells to Matrigel ${ }^{\mathrm{TM}}$. These results indicated that anti-migration, anti-invasion, and anti-adhesion functions may be important contributors to the anti-metastatic activity of ITGB1 inhibition. MMPs are a well-known family of zinc-binding enzymes that have been reported to be upregulated in cancer, and numerous studies have demonstrated that overexpression of MMPs facilitates cancer cell progression, suggesting that MMPs are also involved in metastasis $(31,32)$. In the present study, the inhibition of ITGB1 suppressed MMP-2 and MMP-9 protein expression. These results suggested that ITGB1 inhibition has the potential to inhibit ovarian cancer metastasis by suppression of MMP-2 and MMP-9 expression. In conclusion, inhibition of ITGB1 resulted in tumor cell apoptosis and disrupted tumor mass formation.

Previous studies demonstrated that ITGB1 may be associated with therapeutic resistance to various agents and ionizing radiation in the treatment of cancer (33-36). Notably, ITGB1-mediated resistance is thought to occur at the level of the tumor cells themselves. A recent study demonstrated that ITGB1 inhibition combined with bevacizumab treatment reduced the risk of resistance in glioblastoma (34). In the present study, ITGB1 inhibition enhanced the effects of bevacizumab on apoptosis, adhesion, and migration of ovarian cancer cells. The results lead to the hypothesis that ITGB1 inhibition combined with bevacizumab treatment may reduce the required dose of the bevacizumab anticancer agent, thus potentially reducing drug-related morbidity in ovarian cancer.

It has been suggested that integrin/FAK has an important role in regulating various cellular functions, including adhesion, migration, invasion, survival, growth, and differentiation (37). FAK activates STAT1 in integrin-mediated cell migration and adhesion (29). A previous study demonstrated that FAK/STAT1 increased the malignant potential of ovarian epithelium (28). Therefore, ITGB1/FAK/STAT1 signaling is a promising therapeutic target for ovarian cancer. In the present study the adherence and migratory potentials of ovarian cancer cells were significantly reduced following the inhibition of the ITGB1/FAK/STAT1 signaling pathway by fludarabine. These results revealed that inhibiting FAK/STAT1 signaling exerts anti-metastatic effects on ovarian cancer cells.

These data suggested that ITGB1 inhibition effectively reduced tumorigenesis and disease exacerbation, and 
contributed to bevacizumab-induced anticancer therapy via the FAK/STAT1 signaling pathway. This further elucidated the anti-tumor molecular mechanisms underlying ITGB1 inhibition, suggesting that targeting ITGB1 is a potential strategy for the prevention and treatment of ovarian cancer metastasis.

\section{References}

1. Yang W, Han W, Ye S, Liu D, Wu J, Liu H, Li C and Chen H: Fibroblast activation protein- $\alpha$ promotes ovarian cancer cell proliferation and invasion via extracellular and intracellular signaling mechanisms. Exp Mol Pathol 95: 105-110, 2013.

2. Heyman L, Kellouche S, Fernandes J, Dutoit S, Poulain L and Carreiras F: Vitronectin and its receptors partly mediate adhesion of ovarian cancer cells to peritoneal mesothelium in vitro. Tumour Biol 29: 231-244, 2008.

3. Gotzmann J, Mikula M, Eger A, Schulte-Hermann R, Foisner R, Beug H and Mikulits W: Molecular aspects of epithelial cell plasticity: Implications for local tumor invasion and metastasis. Mutat Res 566: 9-20, 2004.

4. Foubert $P$ and Varner JA: Integrins in tumor angiogenesis and lymphangiogenesis. Methods Mol Biol 757: 471-486, 2012.

5. Garmy-Susini B and Varner JA: Roles of integrins in tumor angiogenesis and lymphangiogenesis. Lymphat Res Biol 6: 155-163, 2008.

6. Tchaicha JH, Reyes SB, Shin J, Hossain MG, Lang FF and McCarty JH: Glioblastoma angiogenesis and tumor cell invasiveness are differentially regulated by $\beta 8$ integrin. Cancer Res 71: 6371-6381, 2011.

7. Buczek-Thomas JA, Chen $\mathrm{N}$ and Hasan T: Integrin-mediated adhesion and signalling in ovarian cancer cells. Cell Signal 10: 55-63, 1998

8. Lau MT, So WK and Leung PC: Integrin $\beta 1$ mediates epithelial growth factor-induced invasion in human ovarian cancer cells. Cancer Lett 320: 198-204, 2012.

9. Tucker GC: Integrins: Molecular targets in cancer therapy. Curr Oncol Rep 8: 96-103, 2006.

10. Cordes N and Park CC: Beta1 integrin as a molecular therapeutic target. Int J Radiat Biol 83: 753-760, 2007.

11. Desgrosellier JS and Cheresh DA: Integrins in cancer: Biological implications and therapeutic opportunities. Nat Rev Cancer 10: 9-22, 2010.

12. Meads MB, Gatenby RA and Dalton WS: Environment-mediated drug resistance: A major contributor to minimal residual disease. Nat Rev Cancer 9: 665-674, 2009.

13. Hazlehurst LA, Landowski TH and Dalton WS: Role of the tumor microenvironment in mediating de novo resistance to drugs and physiological mediators of cell death. Oncogene 22: 7396-7402, 2003.

14. Matsunaga T, Fukai F, Miura S, Nakane Y, Owaki T, Kodama H, Tanaka M, Nagaya T, Takimoto R, Takayama T and Niitsu Y: Combination therapy of an anticancer drug with the FNIII14 peptide of fibronectin effectively overcomes cell adhesion-mediated drug resistance of acute myelogenous leukemia. Leukemia 22: 353-360, 2008.

15. Golubovskaya VM, Kweh FA and Cance WG: Focal adhesion kinase and cancer. Histol Histopathol 24: 503-510, 2009.

16. Lark AL, Livasy CA, Dressler L, Moore DT, Millikan RC, Geradts J, Iacocca M, Cowan D, Little D, Craven RJ and Cance W: High focal adhesion kinase expression in invasive breast carcinomas is associated with an aggressive phenotype. Mod Pathol 18: 1289-1294, 2005.

17. Ucar DA and Hochwald SN: FAK and interacting proteins as therapeutic targets in pancreatic cancer. Anticancer Agents Med Chem 10: 742-746, 2010.

18. Lechertier T and Hodivala-Dilke K: Focal adhesion kinase and tumour angiogenesis. J Pathol 226: 404-412, 2012.
19. Mitra SK and Schlaepfer DD: Integrin-regulated FAK-Src signaling in normal and cancer cells. Curr Opin Cell Biol 18: 516-523, 2006.

20. Jiang J, Grieb B, Thyagarajan A and Sliva D: Ganoderic acids suppress growth and invasive behavior of breast cancer cells by modulating AP-1 and NF-kappaB signaling. Int J Mol Med 21: 577-584, 2008.

21. Bird IM: Extraction of RNA from cells and tissue. Methods Mol Med 108: 139-148, 2005.

22. Song J, Zhang J, Wang J, Cao Z, Wang J, Guo X and Dong W: $\beta 1$ integrin modulates tumor growth and apoptosis of human colorectal cancer. Oncol Rep 32: 302-308, 2014.

23. Liu B, Che W, Xue J, Zheng C, Tang K, Zhang J, Wen J and $\mathrm{Xu}$ Y: SIRT4 prevents hypoxia-induced apoptosis in $\mathrm{H} 9 \mathrm{c} 2$ cardiomyoblast cells. Cell Physiol Biochem 32: 655-662, 2013.

24. Liang CC, Park AY and Guan JL: In vitro scratch assay: A convenient and inexpensive method for analysis of cell migration in vitro. Nat Protoc 2: 329-333, 2007.

25. Monaco S, Gioia M, Rodriguez J, Fasciglione GF, Di Pierro D, Lupidi G, Krippahl L, Marini S and Coletta M: Modulation of the proteolytic activity of matrix metalloproteinase-2 (gelatinase A) on fibrinogen. Biochem J 402: 503-513, 2007.

26. Omanakuttan A, Nambiar J, Harris RM, Bose C, Pandurangan N, Varghese RK, Kumar GB, Tainer JA, Banerji A, Perry JJ, et al: Anacardic acid inhibits the catalytic activity of matrix metalloproteinase-2 and matrix metalloproteinase-9. Mol Pharmacol 82: 614-622, 2012.

27. Tomao F, Papa A, Rossi L, Caruso D, Panici PB, Venezia M and Tomao S: Current status of bevacizumab in advanced ovarian cancer. Onco Targets Ther 6: 889-899, 2013.

28. Zhang L, Wang D, Jiang W, Edwards D, Qiu W, Barroilhet LM, Rho JH, Jin L, Seethappan V, Vitonis A, et al: Activated networking of platelet activating factor receptor and FAK/STAT1 induces malignant potential in BRCA1-mutant at-risk ovarian epithelium. Reprod Biol Endocrinol 8: 74, 2010.

29. Xie B, Zhao J, Kitagawa M, Durbin J, Madri JA, Guan JL and Fu XY: Focal adhesion kinase activates Stat in integrin-mediated cell migration and adhesion. J Biol Chem 276: 19512-19523, 2001.

30. Casey RC and Skubitz AP: CD44 and betal integrins mediate ovarian carcinoma cell migration toward extracellular matrix proteins. Clin Exp Metastasis 18: 67-75, 2000.

31. Mannello F, Tonti G and Papa S: Matrix metalloproteinase inhibitors as anticancer therapeutics. Curr Cancer Drug Targets 5: 285-298, 2005.

32. Radisky ES and Radisky DC: Matrix metalloproteinase-induced epithelial-mesenchymal transition in breast cancer. J Mammary Gland Biol Neoplasia 15: 201-212, 2010.

33. Pontiggia O, Sampayo R, Raffo D, Motter A, Xu R, Bissell MJ, Joffé EB and Simian M: The tumor microenvironment modulates tamoxifen resistance in breast cancer: A role for soluble stromal factors and fibronectin through $\beta 1$ integrin. Breast Cancer Res Treat 133: 459-471, 2012.

34. Carbonell WS, DeLay M, Jahangiri A, Park CC and Aghi MK: $\beta 1$ integrin targeting potentiates antiangiogenic therapy and inhibits the growth of bevacizumab-resistant glioblastoma. Cancer Res 73: 3145-3154, 2013.

35. Kanda R, Kawahara A, Watari K, Murakami Y, Sonoda K, Maeda M, Fujita H, Kage M, Uramoto H, Costa C, et al: Erlotinib resistance in lung cancer cells mediated by integrin $\beta 1 /$ Src/Akt-driven bypass signaling. Cancer Res 73: 6243-6253, 2013.

36. Ju L, Zhou C, Li W and Yan L: Integrin betal over-expression associates with resistance to tyrosine kinase inhibitor gefitinib in non-small cell lung cancer. J Cell Biochem 111: 1565-1574, 2010.

37. Yamamoto N, Kinoshita T, Nohata N, Itesako T, Yoshino H, Enokida H, Nakagawa M, Shozu M and Seki N: Tumor suppressive microRNA-218 inhibits cancer cell migration and invasion by targeting focal adhesion pathways in cervical squamous cell carcinoma. Int J Oncol 42: 1523-1532, 2013. 